\title{
Communicating ASP and the Polynomial Hierarchy
}

\author{
Kim Bauters ${ }^{1, \star}$, Steven Schockaert ${ }^{1, \star \star}$, Dirk Vermeir ${ }^{2}$, and Martine De Cock $^{1}$ \\ 1 Department of Applied Mathematics and Computer Science \\ Universiteit Gent, Krijgslaan 281, 9000 Gent, Belgium \\ \{kim.bauters, steven.schockaert, martine.decock\}@ugent.be \\ 2 Department of Computer Science \\ Vrije Universiteit Brussel, Pleinlaan 2, 1050 Brussel, Belgium \\ dvermeir@vub.ac.be
}

\begin{abstract}
Communicating answer set programming is a framework to represent and reason about the combined knowledge of multiple agents using the idea of stable models. The semantics and expressiveness of this framework crucially depends on the nature of the communication mechanism that is adopted. The communication mechanism we introduce in this paper allows us to focus on a sequence of programs, where each program in the sequence may successively eliminate some of the remaining models. The underlying intuition is that of leaders and followers: each agent's decisions are limited by what its leaders have previously decided. We show that extending answer set programs in this way allows us to capture the entire polynomial hierarchy.
\end{abstract}

\section{Introduction}

Communicating answer set programming is an extension of answer set programming (ASP) in which a number of logic programs, with their own knowledge and reasoning capabilities, can communicate and cooperate with each other to solve the problem at hand. A number of different flavors of Communicating ASP have already been proposed in the literature (e.g. [18/21]). Communicating ASP is also closely related to the research on multi-context systems, where a group of agents can cooperate to find the solution of a global problem [4|20. We start off with an introductory example.

Example 1. An employee (' $E$ ') needs a new printer (' $P$ '). She has a few choices (loud or silent, stylish or dull), preferring silent and stylish. Her boss (' $B$ ') does not want an expensive printer, i.e. one that is both silent and stylish. We can

\footnotetext{
* Funded by a joint Research Foundation-Flanders (FWO) project.

${ }^{\star \star}$ Postdoctoral fellow of the Research Foundation-Flanders (FWO).

J. Delgrande and W. Faber (Eds.): LPNMR 2011, LNAI 6645, pp. 67-79 2011.

(C) Springer-Verlag Berlin Heidelberg 2011
} 
consider the communicating program $\mathcal{P}=\{E, B\}$ with:

$$
\begin{aligned}
P: \text { stylish } & \leftarrow \text { not } P: \text { dull } & P: \text { dull } & \leftarrow \text { not } P: \text { stylish } \\
P: \text { silent } & \leftarrow \text { not } P: \text { loud } & P: \text { loud } & \leftarrow \text { not } P: \text { silent } \\
\text { E: undesired } & \leftarrow P: \text { dull } & \text { E:undesired } & \leftarrow P: \text { loud }
\end{aligned}
$$

Intuitively, the rule ' $B$ :expensive $\leftarrow P$ : stylish, $P$ : silent' expresses that the agent $B$ believes that the printer is 'expensive' when according to agent $P$ it is 'stylish' and 'silent'. The rules in (11) and (2) encode the four possible printers, the rules in (3) and (4) encode the inclinations of the employee and boss, respectively. The answer sets of this program, i.e. those with global minimality, are [1]

$$
\begin{array}{ll}
M_{1}=\{P: \text { sty }, P: \text { silent }, B: \exp \} & M_{2}=\{P: \text { sty }, P: \text { loud }, E: \text { und }\} \\
M_{3}=\{P: \text { dull }, P: \text { loud }, E: \text { und }\} & M_{4}=\{P: \text { dull, } P: \text { silent }, E: \text { und }\}
\end{array}
$$

where for compactness we write $P: s t y, E: u n d$ and $B: \exp$ instead of $P:$ stylish, $E$ : undesired and $B$ :expensive, respectively. The answer sets with minimality for the agent $B$ are $M_{2}, M_{3}$ and $M_{4}$, i.e. the answer sets that do not contain $B$ :expensive. The only answer set with minimality for agent $E$ is $M_{1}$, i.e. the one that does not contain $E$ : undesired. Hence when we determine local minimality for communicating ASP, the order in which we determine the local minimality is important and induces a preference over the agents, i.e. it makes some agents more important than others. In this example, if the boss comes first, the employee no longer has the choice to pick $M_{1}$. This leaves her with the choice of either a dull or a loud printer, among which she has no preferences.

Answer set semantics is based on the idea of stable minimal models. When dealing with agents that can communicate, it becomes unclear how we should interpret the notion of minimality as will become clear later on. One option is to assume global minimality, i.e. we minimize over the conclusions of all the agents in the network. Another option is to assume minimality on the level of a single agent. Since it is not always possible to find a model that is minimal for all individual agents, the order in which we minimize over the agents matters, as the preceding example illustrates.

In this paper we introduce the notion of multi-focused answer sets of communicating ASP programs, which allow us to successively focus (i.e. minimize) on different agents. We study the resulting expressiveness and hence complete the picture sketched in [1 on the effect that adding communication has on the expressiveness of ASP. In [1, it was shown that the ability to communicate allows for simple programs (programs without negation-as-failure, which alone are only capable of expressing problems in P) to simulate normal programs (which do have negation-as-failure and are capable of expressing problems in NP). Furthermore, [1 introduced a new communication mechanism where one can "focus" on a single agent, allowing to express problems in $\Sigma_{2}^{\mathrm{P}}$. In this paper, we go a step further by introducing multi-focused answer sets. Multi-focused answer sets allow us to focus successively on a number of agents instead of focusing on just one 
agent, and is therefore a generalization of the idea of focusing. As it turns out, using multi-focused answer set programs it is possible to express any problem in PSPACE. This means in particular that communicating ASP could be used to solve problems that are above the second level of the polynomial hierarchy, such as some forms of abductive reasoning [10] as well as PSPACE-complete problems such as STRIPS planning $[6$.

The remainder of this paper is organized as follows. In Section 2 we give the necessary background on answer set programming. In Section 3 , we recall the syntax and semantics of communicating ASP. In Section 4 we introduce a generalization of focused answer sets 1], capable of expressing any $\Sigma_{n}^{\mathrm{P}}$ problem. We finish with Section 5 and Section $[$ where we discuss related work and present our conclusion.

\section{Background on Answer Set Programming}

We first recall the basic concepts and results from ASP that are used in this paper. To define ASP programs, we start from a countable set of atoms and we define a literal $l$ as an atom $a$ or its classical negation $\neg a$. If $L$ is a set of literals, we use $\neg L$ to denote the set $\{\neg l \mid l \in L\}$ where, by definition, $\neg \neg a=a$. A set of literals $L$ is consistent if $L \cap \neg L=\emptyset$. An extended literal is either a literal or a literal preceded by not which we call the negation-as-failure operator. Intuitively we say that not $l$ is true when we have no proof to support $l$. For a set of literals $L$, we use $\operatorname{not}(L)$ to denote the set $\{$ not $l \mid l \in L\}$.

A normal rule is an expression of the form $l \leftarrow(\alpha \cup n o t(\beta))$ with ' $l$ ' a literal called the head of the rule and $(\alpha \cup \operatorname{not}(\beta))$ (interpreted as a conjunction) the body of the rule with $\alpha$ and $\beta$ sets of literals. When the body is empty, the rule is called a fact. When the head is empty, the rule is called a constraint. In this paper, we do not consider constraints as they can readily be simulated1. A normal program $P$ is a finite set of normal rules. The Herbrand base $\mathcal{B}_{P}$ of $P$ is the set of atoms appearing in program $P$. A (partial) interpretation $I$ of $P$ is any consistent set of literals $L \subseteq\left(\mathcal{B}_{P} \cup \neg \mathcal{B}_{P}\right)$. $I$ is total iff $I \cup \neg I=\mathcal{B}_{P} \cup \neg \mathcal{B}_{P}$. A simple rule is a normal rule without negation-as-failure in the body. A simple program $P$ is a finite set of simple rules.

Answer sets are defined using the immediate consequence operator $T_{P}$ for a simple program $P$ w.r.t. an interpretation $I$ as

$$
T_{P}(I)=I \cup\{l \mid((l \leftarrow \alpha) \in P) \wedge(\alpha \subseteq I)\} .
$$

We use $P^{\star}$ to denote the fixpoint which is obtained by repeatedly applying $T_{P}$ starting from the empty interpretation, i.e. the least fixpoint of $T_{P}$ w.r.t. set inclusion. The interpretation $P^{\star}$ is the minimal model of $P$ and is called the answer set of the simple program $P$.

The reduct $P^{I}$ of a normal program $P$ w.r.t. the interpretation $I$ is defined as $P^{I}=\{l \leftarrow \alpha \mid(l \leftarrow \alpha \cup n o t(\beta)) \in P, \beta \cap I=\emptyset\}$. It is easy to see that the reduct $P^{I}$ is a simple program. We say that $I$ is an answer set of the normal program $P$ iff $\left(P^{I}\right)^{\star}=I$, i.e. if $I$ is the answer set of the reduct $P^{I}$.

\footnotetext{
${ }^{1}$ The constraint $(\leftarrow$ body $)$ is simulated by $($ fail $\leftarrow$ not fail, body) with fail a fresh atom.
} 


\section{Communicating Programs}

Communication between ASP programs is based on a new kind of literal ' $Q: l$ ' as in [1316 20, where the underlying intuition is that of a function call or, in terms of agents, asking questions to other agents. If the literal $l$ is not in the answer set of program $Q$ then $Q: l$ is false; otherwise $Q: l$ is true. We present the semantics from [1] which are closely related to the minimal semantics in [3] and the semantics in [5].

Let $\mathcal{P}$ be a finite set of program names. A $\mathcal{P}$-situated literal is an expression of the form $Q: l$ with $Q \in \mathcal{P}$ and $l$ a literal. A $\mathcal{P}$-situated literal $Q: l$ is called $Q$-local. For a set of $\mathcal{P}$-situated literals $X$ and $Q \in \mathcal{P}$, we use $X_{\downarrow Q}$ to denote $\{l \mid Q: l \in X\}$, i.e. the projection of $X$ on $Q$. An extended $\mathcal{P}$-situated literal is either a $\mathcal{P}$-situated literal or a $\mathcal{P}$-situated literal preceded by $n o t$. For a set of $\mathcal{P}$-situated literals $X$, we use $\operatorname{not}(X)$ to denote the set $\{$ not $Q: l \mid Q: l \in X\}$.

A $\mathcal{P}$-situated normal rule is an expression of the form $Q: l \leftarrow(\alpha \cup \operatorname{not}(\beta))$ where $Q: l$ is called the head of the rule, and $\alpha \cup \operatorname{not}(\beta)$ is called the body of the rule with $\alpha$ and $\beta$ sets of $\mathcal{P}$-situated literals. A $\mathcal{P}$-situated normal rule $Q: l \leftarrow(\alpha \cup$ not $(\beta))$ is called $Q$-local. A $\mathcal{P}$-component normal program $Q$ is a finite set of $Q$-local $\mathcal{P}$-situated normal rules. Henceforth we shall use $\mathcal{P}$ both to denote the set of program names and to denote the set of actual $\mathcal{P}$-component normal programs. A communicating normal program $\mathcal{P}$ is then a finite set of $\mathcal{P}$-component normal programs. A $\mathcal{P}$-situated simple rule is an expression of the form $Q: l \leftarrow \alpha$, i.e. a $\mathcal{P}$-situated normal rule without negation-as-failure in the body. A $\mathcal{P}$-component simple program $Q$ is a finite set of $Q$-local $\mathcal{P}$-situated simple rules. A communicating simple $\operatorname{program} \mathcal{P}$ is a finite set of $\mathcal{P}$-component simple programs.

In the remainder of this paper we drop the $\mathcal{P}$-prefix whenever the set $\mathcal{P}$ is clear from the context. Whenever the name of the component normal program $Q$ is clear, we write $l$ instead of $Q: l$ for $Q$-local situated literals. Note that a communicating normal (resp. simple) program with only one component program thus trivially corresponds to a normal (resp. simple) program.

Similar as for a classical program, we can define the Herbrand base of a component program $Q$ as the set of atoms occurring in $Q$-local situated literals in $Q$, which we denote as $\mathcal{B}_{Q}$. We then define $\mathcal{B}_{\mathcal{P}}=\left\{Q: a \mid Q \in \mathcal{P}\right.$ and $\left.a \in \bigcup_{R \in \mathcal{P}} \mathcal{B}_{R}\right\}$ as the Herbrand base of the communicating program $\mathcal{P}$.

Example 2. Consider the communicating simple program $\mathcal{P}=\{Q, R\}$ with the following situated rules:

$$
Q: a \leftarrow R: a \quad Q: b \leftarrow \quad R: a \leftarrow Q: a .
$$

$Q: a, Q: b$ and $R: a$ are situated literals. The situated simple rules $Q: a \leftarrow R: a$ and $Q: b \leftarrow$ are $Q$-local since we have $Q: a$ and $Q: b$ in the heads of these rules. The situated simple rule $R: a \leftarrow Q: a$ is $R$-local. Hence $Q=\{a \leftarrow R: a, b \leftarrow\}$ and $R=\{a \leftarrow Q: a\}$. Furthermore, we have that $\mathcal{B}_{Q}=\{a, b\}, \mathcal{B}_{R}=\{a\}$ and $\mathcal{B}_{\mathcal{P}}=\{Q: a, Q: b, R: a, R: b\}$. 
We say that a (partial) interpretation $I$ of a communicating program $\mathcal{P}$ is any consistent subset $I \subseteq\left(\mathcal{B}_{\mathcal{P}} \cup \neg \mathcal{B}_{\mathcal{P}}\right)$. Given an interpretation $I$ of a communicating normal program $\mathcal{P}$, the reduct $Q^{I}$ for $Q \in \mathcal{P}$ is the component simple program obtained by deleting

- each rule with an extended situated literal 'not $R: l$ ' such that $R: l \in I$;

- each remaining extended situated literal of the form 'not $R: l$ ';

- each rule with a situated literal ' $R: l$ ' that is not $Q$-local such that $R: l \notin I$;

- each remaining situated literal $R: l$ that is not $Q$-local.

The underlying intuition of the reduct is clear. Analogous to the definition of the reduct for normal programs [15], the reduct of a communicating normal program defines a way to reduce a program relative to some guess $I$. The reduct of a communicating normal program is a communicating simple program that only contains component simple programs $Q$ with $Q$-local situated literals. That is, each component simple program $Q$ corresponds to a classical simple program.

Definition 1. We say that an interpretation $I$ of a communicating normal program $\mathcal{P}$ is an answer set of $\mathcal{P}$ if and only if $\forall Q \in \mathcal{P} \cdot\left(Q: I_{\downarrow}\right)=\left(Q^{I}\right)^{\star}$.

In other words: an interpretation $I$ is an answer set of a communicating normal program $\mathcal{P}$ if and only if for every component normal program $Q$ we have that the projection of $I$ on $Q$ is an answer set of the component normal program $Q$ under the classical definition.

Example 3. Let us once again consider the communicating simple program $\mathcal{P}=$ $\{Q, R\}$ from Example 2. Given the interpretation $I=\{Q: a, Q: b, R: a\}$ we find that $Q^{I}=\{a \leftarrow, b \leftarrow\}$ and $R^{I}=\{a \leftarrow\}$. We can easily treat $Q^{I}$ and $R^{I}$ separately since they now correspond to classical programs. It then readily follows that $Q: I_{\downarrow Q}=\left(Q^{I}\right)^{\star}$ and $R: I_{\downarrow R}=\left(R^{I}\right)^{\star}$, hence the interpretation $I$ is an answer set of $\mathcal{P}$. In total the communicating simple program $\mathcal{P}$ has two answer sets, namely $\{Q: b\}$ and $\{Q: a, Q: b, R: a\}$.

Proposition 1. 11 Let $\mathcal{P}$ be a communicating simple program. Determining whether a given situated literal $Q: l$ occurs in any answer set of $\mathcal{P}$ (i.e. brave reasoning) is in NP. Determining whether a literal is true in all the answer sets of $\mathcal{P}$ (i.e. cautious reasoning) is in coNP. Furthermore, the same complexity results hold when $\mathcal{P}$ is a communicating normal program.

\section{Multi-focused Answer Sets}

We extend the semantics of communicating programs in such a way that it becomes possible to focus on a sequence of component programs. As such, we can indicate that we are only interested in those answer sets that are successively minimal with respect to each respective component program. The underlying intuition is that of leaders and followers, where the decisions that an agent can make are limited by what its leaders have previously decided. 
Definition 2. Let $\mathcal{P}$ be a communicating program and $\left\{Q_{1}, \ldots, Q_{n}\right\} \subseteq \mathcal{P}$ a set of component programs. $A\left(Q_{1}, \ldots, Q_{n}\right)$-focused answer set of $\mathcal{P}$ is defined as:

$$
\begin{aligned}
& M \text { is a }\left(Q_{1}, \ldots, Q_{n-1}\right) \text {-focused answer set of } \mathcal{P} \text { and } \\
& \quad \text { for each }\left(Q_{1}, \ldots, Q_{n-1}\right) \text {-focused answer set } M^{\prime} \text { of } \mathcal{P} \\
& \quad \text { we do not have that } M^{\prime}{ }_{\downarrow Q_{n}} \subset M_{\downarrow Q_{n}}
\end{aligned}
$$

where a ()-focused answer set of $\mathcal{P}$ is any answer set of $\mathcal{P}$.

In other words, we say that $M$ is a $\left(Q_{1}, \ldots, Q_{n}\right)$-focused answer set of $\mathcal{P}$ if and only if $M$ is minimal among all $\left(Q_{1}, \ldots, Q_{n-1}\right)$-focused answer sets w.r.t. the projection on $Q_{n}$.

Example 4. Consider the communicating program $\mathcal{A}[4]=\{Q, R, S\}$ with the rules

$$
\begin{array}{lll}
Q: a \leftarrow & R: b \leftarrow S: c & S: a \leftarrow \\
Q: b \leftarrow \text { not } S: d & R: a \leftarrow S: c & S: c \leftarrow \text { not } S: d, \text { not } R: c \\
Q: c \leftarrow R: c & R: a \leftarrow S: d & S: c \leftarrow \text { not } S: c, \text { not } R: c \\
& R: c \leftarrow \text { not } R: a &
\end{array}
$$

The communicating program $\mathcal{F}_{4}$ has three answer sets, namely

$$
\begin{aligned}
& M_{1}=Q:\{a, b, c\} \cup R:\{c\} \cup S:\{a\} \\
& M_{2}=Q:\{a, b\} \cup R:\{a, b\} \cup S:\{a, c\} \\
& M_{3}=Q:\{a\} \cup R:\{a\} \cup S:\{a, d\} .
\end{aligned}
$$

The only $(R, S)$-focused answer set of $\mathcal{F}$ 开 is $M_{1}$. Indeed, since $\{a\}=\left(M_{3}\right)_{\downarrow R} \subset$ $\left(M_{2}\right)_{\downarrow R}=\{a, b\}$ we find that $M_{2}$ is not a $(R)$-focused answer set. Furthermore $\{a\}=\left(M_{1}\right)_{\downarrow S} \subset\left(M_{3}\right)_{\downarrow S}=\{a, d\}$, hence $M_{3}$ is not an $(R, S)$-focused answer set.

We now show how the validity of quantified boolean formulas (QBF) can be checked using multi-focused answer sets of communicating ASP programs.

Definition 3. Let $\phi=\exists X_{1} \forall X_{2} \ldots \Theta X_{n} \cdot p\left(X_{1}, X_{2}, \cdots X_{n}\right)$ be a $Q B F$ where $\Theta=\forall$ if $n$ is even and $\Theta=\exists$ otherwise, and $p\left(X_{1}, X_{2}, \cdots X_{n}\right)$ is a formula of the form $\theta_{1} \vee \ldots \vee \theta_{m}$ in disjunctive normal form over $X_{1} \cup \ldots \cup X_{n}$ with $X_{i}, 1 \leq i \leq n$, sets of variables and where each $\theta_{t}$ is a conjunction of propositional literals. We define $Q_{0}$ as follows:

$$
\begin{aligned}
Q_{0}= & \left\{x \leftarrow \text { not } \neg x, \neg x \leftarrow \text { not } x \mid x \in X_{1} \cup \ldots \cup X_{n}\right\} \\
& \cup\left\{\text { sat } \leftarrow Q_{0}: \theta_{t} \mid \theta_{t}, 1 \leq t \leq m\right\} \\
& \cup\{\neg \text { sat } \leftarrow \text { not sat }\} .
\end{aligned}
$$

For $1 \leq j \leq n-1$ we define $Q_{j}$ as follows:

$$
\begin{aligned}
Q_{j}= & \left\{x \leftarrow Q_{0}: x, \neg x \leftarrow Q_{0}: \neg x \mid x \in\left(X_{1} \cup \ldots \cup X_{n-j}\right)\right\} \\
& \cup \begin{cases}\left\{\neg s a t \leftarrow Q_{0}: \neg \text { sat }\right\} & \text { if }(n-j) \text { is even } \\
\left\{\text { sat } \leftarrow Q_{0}: \text { sat }\right\} & \text { if }(n-j) \text { is odd } .\end{cases}
\end{aligned}
$$


The communicating normal program corresponding with $\phi$ is $\mathcal{P}=\left\{Q_{0}, \ldots, Q_{n-1}\right\}$.

For a $Q B F$ of the form $\phi=\forall X_{1} \exists X_{2} \ldots \Theta X_{n} \cdot p\left(X_{1}, X_{2}, \cdots X_{n}\right)$ where $\Theta=\exists$ if $n$ is even and $\Theta=\forall$ otherwise and $p\left(X_{1}, X_{2}, \cdots X_{n}\right)$ once again a formula in disjunctive normal form, the simulation only changes slightly. Indeed, only the conditions in (10) are swapped.

Example 5. Given the QBF $\phi=\exists x \forall y \exists z \cdot(x \wedge y) \vee(\neg x \wedge y \wedge z) \vee(\neg x \wedge \neg y \wedge \neg z)$, the communicating program $\mathcal{P}$ corresponding with the $\mathrm{QBF} \phi$ is defined as follows:

$$
\begin{array}{rlrlrl}
Q_{0}: x & \leftarrow \text { not } \neg x & Q_{0}: y & \leftarrow \text { not } \neg y & Q_{0}: z & \leftarrow \text { not } \neg z \\
Q_{0}: \neg x & \leftarrow \text { not } x & Q_{0}: \neg y & \leftarrow \text { not } y & Q_{0}: \neg z & \leftarrow \text { not } z \\
Q_{0}: \text { sat } & \leftarrow x, y & Q_{0}: \text { sat } & \leftarrow \neg x, y, z & Q_{0}: \text { sat } & \leftarrow \neg x, \neg y, \neg z \\
Q_{0}: \neg \text { sat } & \leftarrow \text { not sat } & & & \\
Q_{1}: x & \leftarrow Q_{0}: x & Q_{1}: y & \leftarrow Q_{0}: y & & \\
Q_{1}: \neg x & \leftarrow Q_{0}: \neg x & Q_{1}: \neg y & \leftarrow Q_{0}: \neg y & Q_{1}: \neg \text { sat } & \leftarrow Q_{0}: \neg \text { sat } \\
Q_{2}: x & \leftarrow Q_{0}: x & Q_{2}: \neg x & \leftarrow Q_{0}: \neg x & Q_{2}: \text { sat } & \leftarrow Q_{0}: \text { sat }
\end{array}
$$

The communicating program in Example[5 can be used to determine whether the QBF $\phi$ is satisfiable. First, note that the rules in (6) generate all possible truth assignments of the variables, i.e. all possible propositional interpretations. The rules in (7) ensure that 'sat' is true exactly for those interpretations that satisfy the formula $p\left(X_{1}, X_{2}, \ldots, X_{n}\right)$, i.e. we check, for this particular assignment of the variables, whether $p\left(X_{1}, \ldots, X_{n}\right)$ is satisfied.

Intuitively, the component programs $\left\{Q_{1}, \ldots, Q_{n-1}\right\}$ successively bind fewer and fewer variables. In particular, focusing on $Q_{1}, \ldots, Q_{n-1}$ allows us to consider the binding of the variables in $X_{n-1}, \ldots, X_{1}$, respectively. Depending on the rules from (10), focusing on $Q_{i}$ allows us to verify that either some or all of the assignments of the variables in $X_{n-j}$ make the formula $p\left(X_{1}, \ldots, X_{n}\right)$ satisfied, given the bindings that have already been determined by the preceding components. We now prove that the $\mathrm{QBF} \phi$ is satisfiable iff $Q_{0}$ :sat is true in some $\left(Q_{1}, \ldots, Q_{n-1}\right)$-focused answer set.

Proposition 2. Let $\phi$ and $\mathcal{P}$ be as in Definition 3. We have that a $Q B F \phi$ of the form $\phi=\exists X_{1} \forall X_{2} \ldots \Theta X_{n} \cdot p\left(X_{1}, X_{2}, \cdots X_{n}\right)$ is satisfiable if and only if $Q_{0}:$ sat is true in some $\left(Q_{1}, \ldots, Q_{n-1}\right)$-focused answer set of $\mathcal{P}$. Furthermore, we have that a QBF $\phi$ of the form $\phi=\forall X_{1} \exists X_{2} \ldots \Theta X_{n} \cdot p\left(X_{1}, X_{2}, \cdots X_{n}\right)$ is satisfiable if and only if $Q_{0}$ : sat is true in all $\left(Q_{1}, \ldots, Q_{n-1}\right)$-focused answer sets of $\mathcal{P}$.

Proof. We give a proof by induction. Assume we have a QBF $\phi_{1}$ of the form $\exists X_{1}$. $p\left(X_{1}\right)$ with $\mathcal{P}_{1}=\left\{Q_{0}\right\}$ the communicating normal program corresponding with $\phi_{1}$ according to Definition 3, If the formula $p_{1}\left(X_{1}\right)$ of the QBF $\phi_{1}$ is satisfiable then we know that there is a ()-focused answer set $M$ of $\mathcal{P}_{1}$ such that $Q_{0}:$ sat $\in$ $M$. Otherwise, we know that $Q_{0}:$ sat $\notin M$ for all ()-answer sets $M$ of $\mathcal{P}_{1}$. Hence the induction hypothesis is valid for $n=1$. 
Assume the result holds for any QBF $\phi_{n-1}$ of the form $\exists X_{1} \forall X_{2} \ldots \Theta X_{n-1}$. $p_{n}\left(X_{1}, X_{2}, \ldots, X_{n-1}\right)$. We show in the induction step that it holds for any QBF $\phi_{n}$ of the form $\exists X_{1} \forall X_{2} \ldots \bar{\Theta} X_{n} \cdot p_{n-1}\left(X_{1}, X_{2}, \ldots, X_{n}\right)$. Let $\mathcal{P}=\left\{Q_{0}, \ldots, Q_{n-1}\right\}$ and $\mathcal{P}^{\prime}=\left\{Q_{0}^{\prime}, \ldots, Q_{n-2}^{\prime}\right\}$ be the communicating normal programs that correspond with $\phi_{n}$ and $\phi_{n-1}$, respectively. Note that the component programs $Q_{2}, \ldots, Q_{n-1}$ are defined in exactly the same way as the component programs $Q_{1}^{\prime}, \ldots, Q_{n-2}^{\prime}$, the only difference being the name of the component programs. What is of importance in the case of $\phi_{n}$ is therefore only the additional rules in $Q_{0}$ and the new component program $Q_{1}$. The additional rules in $Q_{0}$ merely generate the corresponding interpretations, where we now need to consider the possible interpretations of the variables from $X_{n}$ as well. The rules in the new component program $Q_{1}$ ensure that $Q_{1}: x \in M$ whenever $Q_{0}: x \in M$ and $Q_{1}: \neg x \in M$ whenever $Q_{0}: \neg x \in M$ for every $M$ an answer set of $\mathcal{P}$ and $x \in\left(X_{1} \cup \ldots \cup X_{n-1}\right)$. Depending on $n$ being even or odd, we get two distinct cases:

- if $n$ is even, then we have $\left(\right.$ sat $\left.\leftarrow Q_{0}: s a t\right) \in Q_{1}$ and we know that the QBF $\phi_{n}$ has the form $\exists X_{1} \forall X_{2} \ldots \forall X_{n} \cdot p_{n}\left(X_{1}, X_{2}, \ldots, X_{n}\right)$. Let us consider what happens when we determine the $\left(Q_{1}\right)$-focused answer sets of $\mathcal{P}$. Due to the construction of $Q_{1}$, we know that $M_{\downarrow}^{\prime}{ }_{\downarrow Q_{1}} \subset M_{\downarrow Q_{1}}$ can only hold for two answer sets $M^{\prime}$ and $M$ of $\mathcal{P}$ if $M^{\prime}$ and $M$ correspond to identical interpretations of the variables in $X_{1} \cup \ldots \cup X_{n-1}$. Furthermore, $M^{\prime}{ }_{\downarrow} Q_{1} \subset$ $M_{\downarrow Q_{1}}$ is only possible if $Q_{1}:$ sat $\in M$ while $Q_{1}:$ sat $\notin M^{\prime}$.

Now note that given an interpretation of the variables in $X_{1} \cup \ldots \cup X_{n-1}$, there is exactly one answer set for each choice of $X_{n}$. When we have $M^{\prime}$ with $Q_{1}:$ sat $\notin M^{\prime}$ this implies that there is an interpretation such that, for some choice of $X_{n}$, this particular assignment of values of the QBF does not satisfy the QBF. Similarly, if we have $M$ with $Q_{1}:$ sat $\in M$ then the QBF is satisfied for that particular choice of $X_{n}$. Determining $\left(Q_{1}\right)$-focused answer sets of $\mathcal{P}$ will eliminate $M$ since $M_{\downarrow}^{\prime}{ }_{\downarrow Q_{1}} \subset M_{\downarrow Q_{1}}$. In other words, for identical interpretations of the variables in $X_{1} \cup \ldots \cup X_{n-1}$, the answer set $M^{\prime}$ encodes a counterexample that shows that for these interpretations it does not hold that the QBF is satisfied for all choices of $X_{n}$. Focusing thus eliminates those answer sets that claim that the QBF is satisfiable for the variables in $X_{1} \cup \ldots \cup X_{n-1}$. When we cannot find such $M^{\prime}{ }_{\downarrow Q_{1}} \subset M_{\downarrow Q_{1}}$ this is either because none of the interpretations satisfy the QBF or all of the interpretations satisfy the QBF. In both cases, there is no need to eliminate any answer sets. We thus effectively mimic the requirement that the QBF $\phi_{n}$ should hold for $\forall X_{n}$.

- if $n$ is odd, then $\left(\neg s a t \leftarrow Q_{0}: \neg s a t\right) \in Q_{1}$ and we know that the QBF $\phi_{n}$ has the form $\exists X_{1} \forall X_{2} \ldots \exists X_{n} \cdot p_{n}\left(X_{1}, X_{2}, \ldots, X_{n}\right)$. As before, we know that $M_{\downarrow}^{\prime}{ }_{\downarrow} \subset M_{\downarrow} Q_{1}$ can only hold for two answer sets $M^{\prime}$ and $M$ of $\mathcal{P}$ if $M^{\prime}$ and $M$ correspond to identical interpretations of the variables in $X_{1} \cup \ldots \cup X_{n-1}$. However, this time $M^{\prime} \downarrow_{Q_{1}} \subset M_{\downarrow Q_{1}}$ is only possible if $Q_{1}: \neg s a t \in M$ while $Q_{1}: \neg s a t \notin M^{\prime}$.

If we have $M$ with $Q_{1}: \neg s a t \in M$ then the $\mathrm{QBF}$ is not satisfied for that particular choice of $X_{n}$, whereas when $M^{\prime}$ with $Q_{1}: \neg s a t \notin M^{\prime}$ this 
implies that there is an interpretation such that, for some choice of $X_{n}$, this particular assignment of the variables does satisfy the QBF. Determining $\left(Q_{1}\right)$-focused answer sets of $\mathcal{P}$ will eliminate $M$ since $M^{\prime}{ }_{\downarrow} Q_{1} \subset M_{\downarrow Q_{1}}$. For identical interpretations of the variables in $X_{1} \cup \ldots \cup X_{n-1}$, the answer set $M^{\prime}$ encodes a counterexample that shows that for these interpretations there is some choice of $X_{n}$ such that the QBF is satisfied. Focusing thus eliminates those answer sets that claim that the QBF is not satisfiable for the variables in $X_{1} \cup \ldots \cup X_{n-1}$. When we cannot find such $M^{\prime}{ }_{\downarrow Q_{1}} \subset M_{\downarrow Q_{1}}$ this is either because none of the interpretations satisfy the QBF or all of the interpretations satisfy the QBF. In both cases, there is no need to eliminate any answer sets. We effectively mimic the requirement that the $\mathrm{QBF} \phi_{n}$ should hold for $\exists X_{n}$.

For a QBF of the form $\forall X_{1} \exists X_{2} \ldots \Theta X_{n} \cdot p\left(X_{1}, X_{2}, \ldots, X_{n}\right)$, with $\Theta=\exists$ if $n$ is even and $\Theta=\forall$ otherwise, the proof is analogous. In the base case, we know that a QBF $\phi_{1}$ of the form $\forall X_{1} \cdot p\left(X_{1}\right)$ is satisfiable only when for every ()-focused answer set $M$ of $\mathcal{P}_{1}=\left\{Q_{0}\right\}$ we find that $Q_{0}:$ sat $\in M$. Otherwise, we know that there exists some ()-focused answers sets $M$ of $\mathcal{P}_{1}$ such that $Q_{0}$ : sat $\notin M$. Hence the induction hypothesis is valid for $n=1$. The induction step is then entirely analogous to what we have proven before, with the only difference being that the cases for $n$ being even or odd are swapped. Finally, since the first quantifier is $\forall$, we need to verify that $Q_{0}:$ sat is true in every $\left(Q_{1}, \ldots, Q_{n-1}\right)$-focused answer set of $\mathcal{P}$.

Before we discuss the computational complexity, we recall some of the notions of complexity theory. We have $\Sigma_{0}^{\mathrm{P}}=\mathrm{P}$ and $\Sigma_{1}^{\mathrm{P}}=\mathrm{NP}$ where the complexity class $\Sigma_{n}^{\mathrm{P}}=\mathrm{NP}^{\Sigma_{n-1}^{\mathrm{P}}}$ is the class of problems that can be solved in polynomial time on a non-deterministic machine with an $\Sigma_{n-1}^{\mathrm{P}}$ oracle, i.e. assuming a procedure that can solve $\Sigma_{n-1}^{\mathrm{P}}$ problems in constant time [19]. Deciding the validity of a QBF $\phi=\exists X_{1} \forall X_{2} \ldots \Theta X_{n} \cdot p\left(X_{1}, X_{2}, \cdots X_{n}\right)$ is the canonical $\Sigma_{n}^{\mathrm{P}}$-complete problem. Furthermore $\Pi_{n}^{\mathrm{P}}=\operatorname{co}\left(\Sigma_{n}^{\mathrm{P}}\right)$. Deciding the validity of a $\mathrm{QBF} \phi=\forall X_{1} \exists X_{2} \ldots \Theta X_{n}$. $p\left(X_{1}, X_{2}, \cdots X_{n}\right)$ with $\Theta=\forall$ if $n$ is odd and $\Theta=\exists$ otherwise, is the canonical $\Pi_{n}^{\mathrm{P}}$-complete problem.

Corollary 1. Let $\mathcal{P}$ be a communicating program, $Q_{i} \in \mathcal{P}$. The problem of deciding whether $Q_{i}: l \in M$ (brave reasoning) with $M$ a $\left(Q_{1}, \ldots, Q_{n}\right)$-focused answer set of $\mathcal{P}$ is $\Sigma_{n+1}^{\mathrm{P}}$-hard.

Corollary 2. Let $\mathcal{P}$ be a communicating program, $Q_{i} \in \mathcal{P}$. The problem of deciding whether all $\left(Q_{1}, \ldots, Q_{n}\right)$-focused answer sets contain $Q_{i}: l$ (cautious reasoning) is $\Pi_{n+1}^{\mathrm{P}}$-hard.

In addition to these hardness results, we can also establish the corresponding membership results.

Proposition 3. Let $\mathcal{P}$ be a communicating program, $Q_{i} \in \mathcal{P}$. Deciding whether $Q_{i}: l \in M$ with $M$ a $\left(Q_{1}, \ldots, Q_{n}\right)$-focused answer set of $\mathcal{P}$ is in $\Sigma_{n+1}^{\mathcal{P}}$. 
Proof. (sketch) This proof is by induction on $n$. When $n=1$ we guess a $\left(Q_{1}\right)$ focused answer set $M$ of $\mathcal{P}$ in polynomial time and verify that this is indeed a $\left(Q_{1}\right)$-focused answer set in coNP as in classical ASP, i.e. finding a $\left(Q_{1}\right)$-focused answer set is in $\Sigma_{2}^{\mathrm{P}}$. Given an algorithm to compute the $\left(Q_{1}, \ldots, Q_{n-1}\right)$-focused answer sets of $\mathcal{P}$ in $\Sigma_{n}^{\mathrm{P}}$, we can guess a $\left(Q_{1}, \ldots, Q_{n}\right)$-focused answer set and verify there is no $\left(Q_{1}, \ldots, Q_{n}\right)$-focused answer set $M^{\prime}$ of $\mathcal{P}$ such that $M_{\downarrow}^{\prime} Q_{n} \subset M_{\downarrow Q_{n}}$ using a $\Sigma_{n}^{\mathrm{P}}$ oracle, i.e. the algorithm is in $\Sigma_{n+1}^{\mathrm{P}}$.

Now that we have both hardness and membership results, we readily obtain the following corollary.

Corollary 3. Let $\mathcal{P}$ be a communicating normal program, $Q_{i} \in \mathcal{P}$. The problem of deciding whether $Q_{i}: l \in M$ with $M a\left(Q_{1}, \ldots, Q_{n}\right)$-focused answer set of $\mathcal{P}$ is $\Sigma_{n+1}^{\mathrm{P}}$-complete.

The next corollary provides a result for communicating simple programs instead of communicating normal programs.

Corollary 4. Let $\mathcal{P}$ be a communicating simple program, $Q_{i} \in \mathcal{P}$. The problem of deciding whether $Q_{i}: l \in M$ with $M a\left(Q_{1}, \ldots, Q_{n}\right)$-focused answer set of $\mathcal{P}$ is $\Sigma_{n+1}^{\mathrm{P}}$-complete.

\section{Related Work}

A lot of research has been done, for various reasons, on the subject of combining logic programming with the multi-agent paradigm. One reason for such a combination is that logics can be used to describe the (rational) behavior of the agents [9]. Another reason is that it can be used to combine different flavors of logic programming languages [1118. Such an extension of logic programming can be used to externally solve tasks for which ASP is not suited, while remaining in a declarative framework 13 . It can also be used as a form of cooperation, where multiple agents or contexts collaborate to solve a difficult problem 821 . The approach in this paper falls in the last category and is concerned with how the collaboration of different ASP programs affect the expressiveness of the overall system.

Important work has been done in the domain of multi-context systems (MCS) and multi-agent ASP to enable collaboration between the different contexts/ASP programs. We briefly discuss some of the more prominent work in these areas.

The work of 20] discusses an extension of MCSs [16] that allows MCSs to reason about absent information. Each context only has access to a subset of the available information. In order to share this information, an information flow is defined by the system between the different contexts. This idea was later adopted in the ASP community and in our work in particular.

The current paper has the same syntax as [20] but rather different semantics. The semantics in [20] are closely related to the well-founded semantics [14, whereas ours are closer to the spirit of stable models 15. Another point where 
our semantics differ is that the motivation for accepting a literal as being true can be circular if that explanation relies on other component programs. In [5] this circularity is identified as a requirement for the representation of social reasoning.

The work in 4 further extends the work in 20, introducing a multi-context variant of default logic. This extension guarantees that a number of conclusions from default logic come "for free". The paper is the first to offer a syntactical description of the communication rather than a semantic one, making it easier to implement an actual algorithm. Some of interesting applications of contextual frameworks are shown, e.g. information fusion, game theory and social choice.

Along similar lines [3] combines the non-monotonicity from [20] with the heterogeneous approach from [16] into a single framework for heterogenous nonmonotonic multi-context reasoning. The work in $[3$ introduces several notions of equilibria, including minimal and grounded equilibria. In our approach, local reasoning is captured by grounded equilibria (no circularity allowed) while communicating with other component programs is captured by the weaker concept of minimal equilibria. The work in [3] also offers various membership results on checking the existence of an equilibrium. Most notably, [3] is - to the best of our knowledge - the first to explicitly remark that multi-context systems can be non-monotonic even if all the logics in the component programs are monotonic.

We now direct our attention to work done within the ASP community. The ideas presented in this paper are related to HEX programs 12 in which ASP is extended by higher-order predicates and external atoms. Through these external atoms, knowledge can be exchanged with external sources while remaining within the declarative paradigm. Applicability-wise, HEX is proposed as a tool for nonmonotonic semantic web reasoning under the answer set semantics. Because of this setting, HEX is not primarily targeted at increasing the expressiveness, but foremost at extending the applicability and ease of use of ASP.

Two other important works in the area of multi-agent ASP are [8] and [21]. In both 8 and 21] a multi-agent system is developed in which multiple agents communicate with each other. The communication channel is uni-directional, allowing information to be pushed to the next agent. Both approaches use ASP and have agents that are quite expressive in their own right. Indeed, in 8 each agent is an Ordered Choice Logic Program [2] and in [21] each agent uses the extended answer set semantics.

In 8 the agents can communicate with whomever they want and circular communication is allowed (agent $A$ tells $B$ whom tells $A \ldots$ ), which is similar to our approach. However, in 8 only positive information can be shared and the authors do not look at the actual expressiveness of the framework. In 21] Hierarchical Decision Making is introduced where each agent uses the extended answer set semantics. The network is a linear "hierarchical" network and the framework employs the idea of a failure feedback mechanism. Intuitively, this mechanism allows the previous agent in a network to revise his conclusion when it leads to an unresolvable inconsistency for the next agent in the network. It is this mechanism that gives rise to a higher expressiveness, namely $\Sigma_{n}^{\mathrm{P}}$ for 
a hierarchical network of $n$ agents. Our work is different in that we start from normal and simple ASP programs for the agents. Our communication mechanism is also quite simple and does not rely on any kind of feedback. Regardless, we obtain a comparable expressiveness.

We briefly mention [7, in which recursive modular non-monotonic logic programs (MLP) under the ASP semantics are considered. The main difference between MLP and our simple communication is that our communication is parameter-less, i.e. the truth of a situated literal is not dependent on parameters passed by the situated literal to the target component program. Our approach is clearly different and we cannot readily mimic the behavior of the network as presented in [7. Our complexity results therefore do not directly apply to MLPs.

Finally, we like to point out the resemblance between multi-focused answer sets and the work on multi-level linear programming [17. In multi-level linear programming, different agents control different variables that are outside of the control of the other agents, yet are linked by means of linear inequalities (constraints). The agents have to fix the values of the variables they can control in a predefined order, such that their own linear objective function is optimized. Similarly, in communicating ASP, literals belong to different component programs (agents), and their values are linked through constraints, which in this case take the form of rules. Again the agents act in a predefined order, but now they try to minimize the set of literals they have to accept as being true, rather than a linear objective function.

\section{Conclusion}

We have introduced multi-focused answer sets for communicating programs. The underlying intuition is that of leaders and followers, where the choices available to the followers are limited by what the leaders have previously decided. On a technical level, the problem translates to establishing local minimality for some of the component programs in the communicating program. Since in general it is not possible to ensure local minimality for all component programs, an order must be defined among component programs on which to focus. The result is an increase in expressiveness, where the problem of deciding whether $Q_{i}: l \in M$ with $M$ a $\left(Q_{1}, \ldots, Q_{n}\right)$-focused answer set of $\mathcal{P}$ is $\Sigma_{n+1}^{\mathrm{P}}$-complete. In our work we thus find that the choice of the communication mechanism is paramount w.r.t. the expressiveness of the overall system, irrespective of the expressiveness of the individual agents.

\section{References}

1. Bauters, K., Janssen, J., Schockaert, S., Vermeir, D., De Cock, M.: Communicating answer set programs. In: Tech. Comm. of ICLP 2010, vol. 7, pp. 34-43 (2010)

2. Brain, M., De Vos, M.: Implementing OCLP as a front-end for answer set solvers: From theory to practice. In: Proc. of ASP 2005 (2003)

3. Brewka, G., Eiter, T.: Equilibria in heterogeneous nonmonotonic multi-context systems. In: Proc. of AAAI 2007, pp. 385-390 (2007) 
4. Brewka, G., Roelofsen, F., Serafini, L.: Contextual default reasoning. In: Proc. of. IJCAI 2007, pp. 268-273 (2007)

5. Buccafurri, F., Caminiti, G., Laurendi, R.: A logic language with stable model semantics for social reasoning. In: Garcia de la Banda, M., Pontelli, E. (eds.) ICLP 2008. LNCS, vol. 5366, pp. 718-723. Springer, Heidelberg (2008)

6. Bylander, T.: The computational complexity of propositional STRIPS planning. Artificial Intelligence 69, 165-204 (1994)

7. Dao-Tran, M., Eiter, T., Fink, M., Krennwallner, T.: Modular nonmonotonic logic programming revisited. In: Hill, P.M., Warren, D.S. (eds.) ICLP 2009. LNCS, vol. 5649, pp. 145-159. Springer, Heidelberg (2009)

8. De Vos, M., Crick, T., Padget, J., Brain, M., Cliffe, O., Needham, J.: LAIMA: A multi-agent platform using ordered choice logic programming. In: Baldoni, M., Endriss, U., Zhang, S.-W., Torroni, P. (eds.) DALT 2005. LNCS (LNAI), vol. 3904, pp. 72-88. Springer, Heidelberg (2006)

9. Dell'Acqua, P., Sadri, F., Toni, F.: Communicating agents. In: Proc. of MASL 1999 (1999)

10. Eiter, T., Gottlob, G.: The complexity of logic-based abduction. Journal of the ACM 42, 3-42 (1995)

11. Eiter, T., Ianni, G., Lukasiewicz, T., Schindlauer, R., Tompits, H.: Combining answer set programming with description logics for the semantic web. Artifial Intelligence 172(12-13), 1495-1539 (2008)

12. Eiter, T., Ianni, G., Schindlauer, R., Tompits, H.: A uniform integration of higherorder reasoning and external evaluations in answer-set programming. In: Proc. of IJCAI 2005, pp. 90-96 (2005)

13. Eiter, T., Ianni, G., Schindlauer, R., Tompits, H.: dlvhex: A tool for semantic-web reasoning under the answer-set semantics. In: Proc. of ALPSWS 2006, pp. 33-39 (2006)

14. Gelder, A.V., Ross, K.A., Schlipf, J.S.: The well-founded semantics for general logic programs. Journal of the ACM 38(3), 620-650 (1991)

15. Gelfond, M., Lifzchitz, V.: The stable model semantics for logic programming. In: Proc. of ICLP 1988, pp. 1081-1086 (1988)

16. Giunchiglia, F., Serafini, L.: Multilanguage hierarchical logics or: How we can do without modal logics. Artifial Intelligence 65(1), 29-70 (1994)

17. Jeroslow, R.: The polynomial hierarchy and a simple model for competitive analysis. Mathematical Programming 32, 146-164 (1985)

18. Luo, J., Shi, Z., Wang, M., Huang, H.: Multi-agent cooperation: A description logic view. In: Lukose, D., Shi, Z. (eds.) PRIMA 2005. LNCS, vol. 4078, pp. 365-379. Springer, Heidelberg (2009)

19. Papadimitriou, C.: Computational complexity. Addison-Wesley, Reading (1994)

20. Roelofsen, F., Serafini, L.: Minimal and absent information in contexts. In: Proc. of IJCAI 2005, pp. 558-563 (2005)

21. Van Nieuwenborgh, D., De Vos, M., Heymans, S., Hadavandi, E.: Hierarchical decision making in multi-agent systems using answer set programming. In: Inoue, K., Satoh, K., Toni, F. (eds.) CLIMA 2006. LNCS (LNAI), vol. 4371, pp. 20-40. Springer, Heidelberg (2007) 